\title{
EVALUACIÓN FISIOLÓGICA DE POSTULANTES A BRIGADISTAS FORESTALES COMO PROCESO PREVENTIVO EN SEGURIDAD Y SALUD OCUPACIONAL
}

\section{PHYSIOLOGICAL EVALUATION OF APPLICANTS TO FOREST FIRE FIGHTERS AS PREVENTIVE PROCESS OF OCCUPATIONAL SAFETY AND HEALTH}

\author{
Silvia Lagos Padilla ${ }^{*}$ \\ Alda Orellana Yáñez ${ }^{* *}$ \\ Elias ApUd SimON ${ }^{* * *}$
}

\begin{abstract}
RESUMEN
El objetivo del presente estudio es analizar la importancia de evaluar la aptitud física de las personas que postulan al cargo de brigadista para el combate de incendios forestales. Esta actividad es de altas demandas fisiológicas, genera fatiga y deshidratación provocando en casos extremos golpe de calor y muerte. La población estudiada correspondió a 1.431 varones entre 18 y 29 años de edad, evaluados en la Unidad de Ergonomía de la Universidad de Concepción entre los años 2001 y 2006. Los índices fisiológicos considerados fueron porcentaje corporal de masa grasa y capacidad aeróbica expresada en litros de oxígeno por minuto y en mililitros de oxígeno por minuto y por kilo de peso corporal. Los resultados mostraron que un 7,8\% de los postulantes no tenía la aptitud física mínima para realizar esta actividad; que un 56,7\% excedía los límites de masa grasa recomendados y que sólo un 34,3\% de los postulantes cumplía con los tres requerimientos fisiológicos de referencia. En conclusión, cuando se establecen criterios para trabajos físicamente demandantes y se cuenta con técnicas de evaluación de fácil aplicación, no invasivas y de bajo costo, se dispone de una herramienta de prevención en seguridad y salud ocupacional, que entrega elementos de juicio para proteger y no exponer a trabajadores a labores cuyas exigencias sobrepasan sus capacidades.
\end{abstract}

Palabras claves: Salud ocupacional, seguridad, evaluación fisiológica, brigadistas forestales.

\begin{abstract}
The purpose of the present study is to analyze the importance of physiological methods as preventive tool for the protection of the applicants for the work of forest fire fighters. This activity demands heavy physical work, leading to fatigue and dehydration, and in extreme cases to heat stroke and death. The population studied corresponds to 1431 males from 18 to 29 years of age. They were evaluated at the Unit of Ergonomics of the University of Concepcion between 2001 and 2006. The physiological variables considered were body fat percentage and aerobic capacity, expressed as liters of oxygen per minute and milliliters of oxygen per minute and per kilogram of body weight. The results showed that $7.8 \%$ didn't have the minimum physical fitness aptitude required to carry out this activity, $56,7 \%$ exceeded the limits of body fat and only $34,3 \%$ of the applicants fulfilled the three physiological criteria taken as reference. In conclusion, criteria for safety performance of heavy manual work and the availability of simple, non-invasive and low cost techniques for the evaluation of workers are powerful toolsfor the development of preventive actions to protect safety and health of the working population.
\end{abstract}

Keywords: Occupational health, safety, physiological evaluation, forest fire brigades.

Fecha recepción:02/12/08 Fecha aceptación: 02/04/09

\footnotetext{
* Ingeniero Civil Industrial, profesor asistente Universidad de Concepción, Concepción, Chile. E-mail: slagos@udec.cl

${ }^{*}$ Enfermera, profesor asistente Universidad de Concepción, Concepción, Chile. E-mail: aorellan@udec.cl

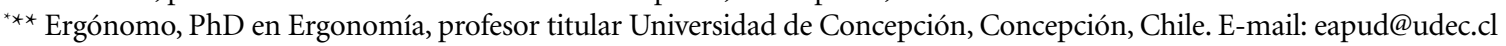




\section{INTRODUCCIÓN}

En Chile, el sector forestal es muy susceptible al daño por incendios, lo que constituye una seria debilidad para el sector (1). Debido a esto, las empresas orientadas a la explotación forestal contratan alrededor de 4.000 trabajadores a comienzos de cada temporada y por los meses de octubre a marzo, para enfrentar los 5.500 incendios rurales que en promedio se producen cada temporada y que destruyen alrededor de 53.000 hectáreas de terreno forestal (2), con daño al patrimonio (3), al medio ambiente $(4,5)$ y con riesgo de accidentes y pérdida de vida de los trabajadores que combaten el fuego (6).

La actividad de brigadista o combatiente forestal es una labor que se realiza en terrenos donde las variables de tipo geográfico y ambiental dificultan la actividad humana. Los brigadistas se enfrentan a fuertes exigencias fisiológicas derivadas del alto gasto de energía que demanda construir cortafuegos con herramientas manuales simples, en terrenos escabrosos, expuestos a gases emitidos por la combustión y a calor radiante, que en un incendio declarado puede alcanzar los $90^{\circ} \mathrm{C}$. Se genera fatiga y deshidratación, pudiendo en casos extremos llevar a los trabajadores al golpe de calor y a la muerte (6). Al respecto, estadísticas del Departamento Forestal de los Estados Unidos revelan que entre 1990 y 1998 murieron 28 brigadistas por problemas cardiacos, debido a las altas demandas fisiológicas impuestas por la actividad (7). Comparativamente, en Chile la tasa de fatalidad en el combate del fuego es un $24,6 \%$ superior a la tasa de fatalidad del combate del fuego en Estados Unidos (2). Se debe considerar, además, que un incendio forestal es una condición de emergencia, un evento no deseado, no planificado, con variables fuera de control y eminentemente peligroso, por lo que se requieren trabajadores con aptitud física por sobre la población promedio para enfrentar de buena forma la tarea (6).
En esta concepción, la Ergonomía es una ciencia aplicada (8), que uniendo profesionales del área de la salud, de la ingeniería y de las ciencias sociales, avanza en la generación de metodologías de investigación, que permiten estudiar la relación entre el bienestar laboral, la salud ocupacional y la productividad $(9,10,11,12)$. Es así como el proyecto FONDEF D99I1072 "Aplicaciones ergonómicas para el aumento de la eficiencia operacional en el combate de incendios forestales" (13), generó recomendaciones basadas en criterios médicos, fisiológicos y psicológicos para la selección de brigadistas forestales, en el entendido que quienes se enfrentan a esta compleja tarea deben ser personas sanas y tener las aptitudes físicas y psicológicas para desempeñar este trabajo (6). Con respecto a los criterios fisiológicos, diversos autores concuerdan en que el grado de condición física y la composición corporal son potentes indicadores del estado de salud de las personas en todas las edades $(14,15)$. Más aún, el proyecto FONDEF D96I1108 (16) mostró que la capacidad aeróbica y la composición corporal son dos indicadores importantes para evaluar la condición física de trabajadores que realizan trabajo dinámico (17). Un buen nivel de capacidad aeróbica está relacionado con la salud cardiovascular y en consecuencia con la capacidad para mantener una tarea físicamente demandante (18). La capacidad aeróbica es el consumo máximo de oxígeno $\left(\mathrm{VO}_{2}\right.$ máx.) que se puede alcanzar durante un ejercicio exhaustivo (19). Refleja la capacidad combinada de los sistemas respiratorio y cardiovascular para obtener, transportar y entregar oxígeno a los músculos durante el trabajo, como también la eficiencia para utilizar oxígeno durante los procesos metabólicos que conducen a la generación de energía. Esta variable se expresa habitualmente en litros de oxígeno consumidos por minuto $\left(\mathrm{l}_{2} \mathrm{~min}^{-1}\right)$ y por mililitros de oxígeno por minuto por kilogramo de peso corporal $\left(\mathrm{ml} \mathrm{O}_{2} \mathrm{~min}^{-1} \mathrm{~kg}^{-1}\right)$. Se utiliza la segunda forma de expresión, porque 
mientras mayor es el peso corporal de una persona, mayor es su gasto de energía en actividades que requieren desplazarlo (6). Cabe destacar que un litro de oxígeno consumido equivale a un gasto energético cercano a cinco kilocalorías. Esto significa que en esfuerzo máximo, un sujeto en los límites más bajos de capacidad aeróbica genera una cantidad muy inferior de energía que los que se ubican en el rango superior $y$, por lo tanto, para igual trabajo, las personas de baja capacidad aeróbica presentan una sobrecarga mayor que los lleva a la fatiga en menor tiempo (12). Esto se ve agravado con el sobrepeso, particularmente en actividades que requieren desplazamiento de la masa corporal (6). En general, la mayor parte de la población masculina tiene capacidades aeróbicas que oscilan entre 2.0 y $4.0 \mathrm{l} \mathrm{O}_{2} \mathrm{~min}^{-1}$ y entre 35 y $55 \mathrm{ml} \mathrm{O}_{2} \mathrm{~min}^{-1} \mathrm{~kg}^{-1}$ (17). Para población forestal, la cuantificación de capacidad aeróbica, expresada en $1 \mathrm{O}_{2} \mathrm{~min}^{-1}$ se muestra en la Tabla 1 (17). Con estos antecedentes, en 2002, Apud y colaboradores (6) recomiendan 3.01 $\mathrm{O}_{2} \mathrm{~min}^{-1}$ como límite inferior de capacidad aeróbica para brigadistas forestales. Cuando se expresa por kilogramo de peso corporal, los mismos autores recomiendan $43,5 \mathrm{ml} \mathrm{O}_{2}$ $\min ^{-1} \mathrm{~kg}^{-1}$, valor que corresponde al prome- dio para población forestal descontada media desviación estándar. A este respecto, valores de consumo de oxígeno publicados por The Cooper Institute for Aerobic Research, sitúan el umbral de salud aeróbica en $42 \mathrm{ml}$ $\mathrm{O}_{2} \mathrm{~min}^{-1} \mathrm{~kg}^{-1}$ para varones (20). Por su parte, las características de una persona en cuanto al contenido corporal de masa grasa (MG), permite una clasificación en los siguientes términos: físico delgado entre 5 y $10 \%$ de grasa corporal, físico corriente entre 10.1 y $15 \%$, sobrepeso entre 15.1 y $20 \%$, obeso superior a $20 \%(6,21,22)$. En este caso y por tratarse de una labor de alta demanda física, en 2002, Apud y colaboradores establecen como criterio que postulantes y brigadistas activos, cuyas edades fluctúen entre 18 y 29 años, no deberían tener más de $15 \%$ de MG (6).

En este contexto, el presente estudio tiene como objetivo analizar la importancia de evaluar la aptitud física de las personas que postulan al cargo de brigadista forestal para el combate de incendios, como herramienta preventiva en la detección de estas, con una aptitud física insuficiente para desempeñar una actividad de alta demanda fisiológica y desarrollada en condiciones ambientales extremas.

Tabla 1: Clasificación para capacidad aeróbica expresada en $1 \mathrm{O}_{2} \min ^{-1}$

\begin{tabular}{|c|c|}
\hline Cap. Aeróbica $\left(\mathbf{I t} \mathbf{~}_{\mathbf{2}} \mathbf{m i n}^{-1}\right.$ ) & Clasificación \\
\hline$<=2.6$ & Baja \\
\hline $2,7-2.9$ & Regular \\
\hline $3.0-3.7$ & Buena \\
\hline $3.8-4.1$ & Muy buena \\
\hline$>=4.2$ & Sobresaliente \\
\hline
\end{tabular}

\section{MATERIAL Y MÉTODO}

La investigación es de tipo longitudinal, descriptiva y prospectiva (23). Los sujetos corresponden al total de postulantes a brigadistas forestales entre 18 y 29 años, que las empresas forestales envían a la Unidad de Ergonomía de la Universidad de Concepción, para realizar pruebas médicas, fisiológicas y psicológicas. Las evaluaciones fisiológicas 
se realizaron en condiciones de laboratorio, con temperatura ambiente menor a $20^{\circ} \mathrm{C}$, con posterioridad a un examen médico y la correspondiente autorización del médico a cargo y con el consentimiento de cada postulante. El año 2001 se evaluaron 197 postulantes, el año 2002, 362; el año 2003, 378; el año 2004, 291; el año 2005, 336; y el año 2006, 52 postulantes.

La capacidad aeróbica se estimó mediante el Nomograma de Astrand y Rhyming, método que requiere someter a la persona a un esfuerzo en que se mide carga de trabajo y frecuencia cardiaca (24). Las pruebas de esfuerzo fueron realizadas en bicicletas ergométricas Cardio Care 828 E, Monark Exercise AB, Suecia. La frecuencia cardiaca se midió con monitores de ritmo cardiaco Polar Vantage NV, Finland (6).

Para evaluar la composición corporal se usó un modelo de dos compartimientos, el cual divide al cuerpo en masa grasa (MG) y masa corporal libre de grasa (MCLG) (25, 26). La MG se estimó a partir de la medición de cuatro pliegues de grasa subcutánea, ubicados en la región bicipital, tricipital, subescapular y suprailíaca. Para la ubicación anatómica de los pliegues, al igual que para el cálculo de la densidad corporal, se utilizó los criterios publicados en 1974 por Durnin \& Womersley (21). Esta técnica fue validada para población chilena en 1980 por Apud \& Jones (22). Los panículos adiposos se midieron con un plicómetro (Holtain LTD. Crymmych UK).

El peso de los postulantes se registró con una balanza DETECTO (DETECTO SCALES INC., Broolyn, NY, USA) y la estatura se midió con un estadiómetro Holtain (Holtain Ltd, Croswell, Crymmych, Pembs.,UK). Estas variables antropométricas se midieron de acuerdo a la técnica descrita por Durnin en 1989 (28).

Los datos se analizaron en el paquete estadístico ISBN 1-884233-49-X (Statistica System for Windows, 2001). Se utilizó estadística descriptiva para caracterizar las variables fisiológicas y los resultados de los análisis de cada una de las variables se reportan como porcentaje, media y desviación estándar.

\section{RESULTADOS}

La edad, peso, talla, capacidad aeróbica y composición corporal de los postulantes a brigadistas forestales estudiados mostró que en promedio tenían buena capacidad aeróbica y por su contenido corporal de masa grasa, un leve sobrepeso. Por su parte, los valores extremos de las variables mostraron personas con baja capacidad aeróbica y un contenido corporal de masa grasa que los calificaba como obesos.

El resultado de segmentar la población en postulantes que alcanzaban los criterios fisiológicos recomendados (Grupo 1) y postulantes que no los alcanzaban (Grupo 2), tal como se muestra en las tablas 2, 3 y 4, mostró que el requisito mínimo de capacidad aeróbica no lo superaron 275 personas, cuando se expresó en $1 \mathrm{O}_{2} \mathrm{~min}^{-1}$ $(19,22 \%)$ y 211 personas $(14,74 \%)$ cuando se expresó en $\mathrm{ml} \mathrm{O}_{2} \mathrm{~min}^{-1} \mathrm{~kg}^{-1}$. Por otra parte, 812 postulantes tenían más de $15 \%$ de masa grasa, lo que equivale al $56,74 \%$ de la población.

Los resultados sobre cuántos criterios fisiológicos superaban los postulantes se presentan en la Tabla 5. Se destaca que un $34,3 \%$ calificó en los tres requisitos fisiológicos solicitados. En el otro extremo, un $7,8 \%$ de los postulantes no superó ninguno de los criterios fisiológicos de referencia.

En el Gráfico 1 se muestra, además, el porcentaje de postulantes que no alcanzaron los mínimos requeridos por criterio y por año. Se aprecia claramente que el porcentaje de masa grasa es el indicador que todos los años presentó mayor incumplimiento, muy por sobre la capacidad aeróbica. 
Tabla 2: Capacidad Aeróbica de los postulantes a brigadistas forestales expresada en $\left(\mathrm{l} \mathrm{O}_{2}\right.$ $\min ^{-1}$ ) y separada en Grupos 1 y 2.

\begin{tabular}{|c|c|c|c|c|c|}
\hline $\begin{array}{c}\text { Cap. Aeróbica } \\
\left(\mathrm{IO}_{2} \min ^{-1}\right) \\
\text { Grupo }\end{array}$ & $\mathrm{N}$ & Means & Std.Dev. & Minimum & Maximum \\
\hline 1 & 1156 & 3,75 & 0,49 & 3,05 & 5,72 \\
\hline 2 & 275 & 2,75 & 0,24 & 1,75 & 3,04 \\
\hline$\overline{\text { Total }}$ & 1431 & & & & \\
\hline
\end{tabular}

Tabla 3: Capacidad Aeróbica de los postulantes a brigadistas forestales entre los años 2001 y 2006 expresada en $\left(\mathrm{ml} \mathrm{O}_{2} \mathrm{~min}^{-1} \mathrm{~kg}^{-1}\right)$ y separada por Grupos 1 y 2.

\begin{tabular}{|c|r|r|r|r|r|}
\hline $\begin{array}{c}\text { Cap. Aeróbica } \\
\left(\mathrm{ml} \mathrm{O}_{2}^{\text {min }^{-1}}\right. \\
\text { Grupo }\end{array}$ & $\mathrm{Ng}$ & Means & Std.Dev. & Minimum & Maximum \\
\hline 1 & 1220 & 55,33 & 7,24 & 43,55 & 90,65 \\
\hline 2 & 211 & 39,42 & 3,60 & 26,41 & 43,48 \\
\hline \hline Total & 1431 & & & & \\
\hline
\end{tabular}

Tabla 4: Porcentaje de Masa Grasa de los postulantes a brigadistas forestales entre los años 2001 y 2006 separada por Grupos 1 y 2

\begin{tabular}{|c|r|r|r|r|r|}
\hline $\begin{array}{c}\text { \%Grasa } \\
\text { Grupo }\end{array}$ & $\mathrm{N}$ & \multicolumn{1}{|c|}{ Means } & Std.Dev. & \multicolumn{1}{c|}{ Minimum } & Maximum \\
\hline 1 & 619 & 11,96 & 1,77 & 4,41 & 14,53 \\
\hline 2 & 812 & 18,52 & 2,95 & 14,56 & 30,90 \\
\hline \hline Total & 1431 & & & & \\
\hline
\end{tabular}

Tabla 5: Número de postulantes a brigadistas y porcentaje de cumplimiento de los criterios fisiológicos evaluados durante los años 2001 a 2006.

\begin{tabular}{|l|r|r|}
\hline \multicolumn{1}{|c|}{ Cumple Criterios } & $\mathrm{N}$ & \multicolumn{1}{c|}{$\%$} \\
\hline Cumplen los 3 criterios & 491 & 34,3 \\
\hline Cumplen con 2 criterios & 694 & 48,5 \\
Cumplen con 1 criterio & 134 & 9,4 \\
\hline Cumplen con 0 criterios & 112 & 7,8 \\
\hline \hline All Grps & 1431 & 100,0 \\
\hline
\end{tabular}


Grafico 1: Curvas de los porcentajes de no cumplimiento de los tres criterios fisiológicos utilizados en la evaluación de postulantes a brigadistas forestales durante los años 2001 a 2006

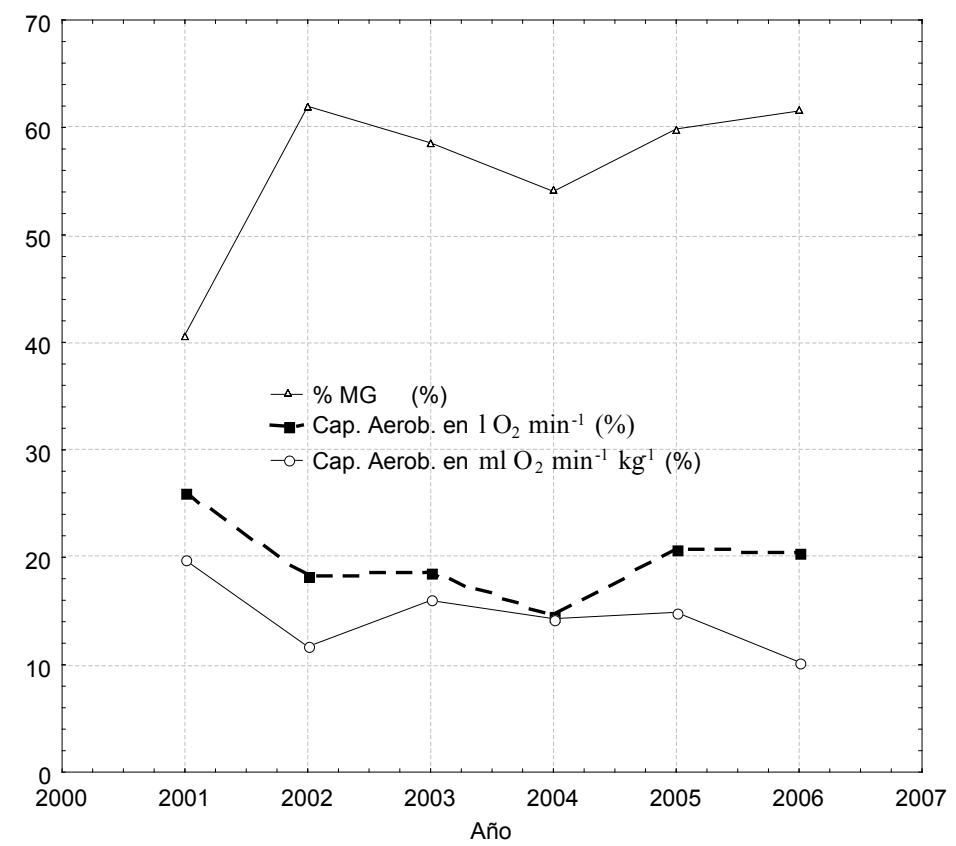

\section{DISCUSIÓN Y CONCLUSIONES}

La población estudiada correspondió a un grupo de varones jóvenes, con peso y estatura dentro del perfil de los trabajadores forestales chilenos (17), con buena condición física y comparativamente superior a otros grupos laborales del país (29). No obstante, debido al porcentaje promedio de masa grasa presentan sobrepeso. Aunque leve, este "sobrepeso" corresponde a un nivel superior al descrito anteriormente para trabajadores forestales chilenos, que en variadas publicaciones han sido clasificados como "corrientes" (10,17,30,31). Este aumento del promedio de grasa corporal concuerda con los resultados de la Cumbre de Nutrición y Salud - Chile 2008 y la tendencia mundial sobre el aumento del contenido corporal de masa grasa en la población (32).

Por otra parte, se destaca que el 7,8\% de los postulantes no presentó la aptitud física necesaria para laborar en condiciones extremas, puesto que todos sus índices fisiológicos escaparon a los criterios de seguridad fijados para esta exigente actividad. A este respecto, los resultados de una evaluación fisiológica no son estáticos. La capacidad aeróbica se incrementa con la actividad física y la composición corporal mejora a través de la disminución del aporte calórico en la ingesta de alimentos, el aumento del gasto de energía, o a la combinación de ambos. De esta forma, una persona que no superó las pruebas de aptitud física, podría hacerlo en una posterior evaluación. Estudios muestran que en un periodo de tres meses, con entrenamiento diario y dieta apropiada, las personas mejoran sustancialmente sus índices fisiológicos (6).

En otro aspecto, las evaluaciones de la aptitud física de trabajadores, para actividades de altas demandas fisiológicas, no es una práctica habitual en nuestro país y cuando se realizan, no hay uniformidad respecto a los criterios que se deben utilizar. En este escenario, el estudio revela la importancia de realizar pruebas de aptitud física en actividades de altas demandas fisiológicas, como herramienta preventiva de salud y seguridad ocupacional. Si las evaluaciones que sirvieron de base para 
el estudio no se hubieran realizado, hipotéticamente, habrían estado integrando una cuadrilla forestal de combate de incendios 112 personas que no tenían, al momento de la evaluación, la aptitud física para hacerlo, aumentando el nivel de riesgo de la actividad. Por este motivo, es imprescindible promover una cultura preventiva, que hasta el momento es considerada como una carencia básica y decisiva en la capacidad de enfrentar los riesgos laborales (33). Por lo expuesto, masificar estas técnicas de fisiología del trabajo puede contribuir a entregar herramientas que potencien la promoción e investigación en prevención. Para esto, es necesario principalmente:

- Que los procedimientos a que son sometidos los trabajadores sean estandarizados, no invasivos y de costo asumible por las empresas.

- Que se evalúe primeramente la carga fisiológica que el trabajo impone (realidad externa), para luego establecer las aptitudes físicas mínimas necesarias que deben cumplir los trabajadores (realidad interna). Todo tendiente a lograr un equilibrio que minimice el riesgo de accidentes y fatalidad de la población laboral.

- Que se fortalezca la formación de competencias en temas de salud laboral y fisiología del trabajo. Esto debido a que los profesionales del área de la salud atienden trabajadores que, en muchos casos, presentan patologías que pueden ser desencadenadas o agravadas por su trabajo (34). Más aún, se reconoce que los accidentes y enfermedades producidos a consecuencia del trabajo son un problema prioritario (35). Pese a esto, en materia de formación y educación, la salud ocupacional aún no se ha posicionado en la práctica cotidiana de los centros de formación profesional (35). En este sentido, esta área se presenta como un potencial campo de desarrollo para los profesionales de la salud.

Finalmente, los resultados de este estudio son aplicables a otras actividades laborales críticas. El trabajo que se realiza en ambientes extremos, de baja o nula posibilidad de modificación y en condiciones de emergencia, no es una situación exclusiva de los brigadistas forestales. Condición que también se identifica en el caso del cuerpo de bomberos, brigadas industriales de emergencia y cuerpos policiales.

\section{REFERENCIAS BIBLIOGRAFICAS}

1. Raga F. El sector forestal chileno y el proyecto país del Colegio de Ingenieros. Santiago, Universidad de Chile, 2004.

2. Ackerknecht C, Mendoza S. Estudio sobre seguridad y salud ocupacional en el combate de incendios forestales en Chile. En Seguridad y salud ocupacional en Chile. Sesión Temática 6, Santiago, 2007. 8 p.

3. Ruiz del Castillo J. Ecología del fuego. En Vélez R. La defensa contra incendios forestales: fundamentos y experiencia. Madrid: McGraw-Hill; 2000. p. 42-65.

4. UNEP. Consideration of global measures on persistent organic pollutants (POPs). Meeting of Government-Designated experts to review and revise a global program of action to protect the marine environment from land-based activities. 1995. UNEP/ ICL/1/Inf 3. No 95-5040.

5. Peña E. Uso del fuego como quema prescrita. Apuntes de clases Ingeniería Forestal. Concepción: Universidad de Concepción; 2000.

6. Apud E, Meyer F, Maureira F. Ergonomía en el Combate de Incendios Forestales, Concepción. Ed. Valverde, 2002.

7. California FACE Report. Fatality Assessment and Control Evaluation Program. 2001. Report 97CA010.

8. Echeverría R. La empresa emergente. Bs. Aires: Granica; 2000.

9. Apud E, Valdés S. Ergonomics in forest: The chilean case. OIT International Labour Office, Switzerland: ILO; 1995. 
10. Apud E, Gutiérrez M, Lagos S, Maureira F. Bases para la certificación de aptitudes físicas, psicológicas y técnicas de trabajadores forestales. Concepción: Fundación Chile; 1996.

11. Apud E, Maureira F, Lagos S, Gutiérrez M. Condiciones laborales y productividad de la mano de obra femenina en la región del Bío-Bío. Concepción: SERNAM; 1999.

12. Apud E, Gutiérrez M, Maureira F, Lagos S, Meyer F, Chiang M. Guía para la evaluación de trabajo pesado: con especial referencia a sobrecarga física y ambiental. Concepción: Publicaciones Universidad de Concepción; 2002.

13. Apud E, Lecannelier E, Gutiérrez M, Lagos S, Maureira, F. Aplicaciones ergonómicas para el aumento de la eficiencia operacional en el combate de incendios forestales. Santiago: FONDEF; 1999. FONDEF D99I1072.

14. Wilmore J, Costill D. Fisiología del esfuerzo y del deporte. Barcelona: Paidotribo; 2004.

15. Casajús J, Leiva M, Ferrando J, Moreno L, Aragonés M, Ara I. Relación entre la condición física cardiovascular y la distribución de grasa en niños y adolescentes. Apunts. Med de l'Esport [en línea] 2006 [fecha de acceso 11 de febrero de 2008]; 41: 7-14. Disponible en: http://www.apunts.org/apunts_ cat/ctl_servlet?_f $=60$ \&ident $=13089453$

16. Apud E, Cortés M, Gutiérrez M, Lagos S, Maureira F, Carmona J, Niebuhr S. Desarrollo y transferencia de tecnologías ergonómicamente adaptadas para el aumento de la productividad del trabajo forestal. Santiago: FONDEF; 1996. FONDEF D96I1108.

17. Apud E, Gutiérrez M, Lagos S, Maureira F, Meyer F, Espinoza J. Manual de Ergonomía Forestal. Concepción: Publicaciones Universidad de Concepción; 1999.

18. Mora M, Mora J, González J, Faraldo F. Valoración de las mejoras provocadas en la capacidad aeróbica en mujeres sedentarias tras un programa de ejercicios. Rev Inter de Med y Cs de la Act Física y el Deporte. 2005; 5 (17): 39-49.

19. Garrido R, González M. Volumen de oxígeno por kilogramo de masa muscular en futbolistas. Rev Inter de Med y Cs de la Act Física y el Deporte. 2006; 6 (21): 44-61.

20. The Cooper Institute for Aerobic Research, FITNESSGRAM test administration manual. Champaign: Human Kinetics. 1999; 38-9.

21. Durnin J, Womersley J. Body fat assessed from total body density and its estimation from skinfold thickness: measurements on 481 men and women aged from $16-72$ years. Br J Nutr. 1974; 32:77-96.

22. Apud E, Jones P. Validez de la medición de los pliegues de grasa subcutánea en estudios de composición corporal. Rev Médica de Chile. 1980; 101: 661-673.

23. Pineda E, De Albarado E. Metodología de la Investigación. $3^{\text {a }}$ ed. Washington DC: OPS; 2008.

24. Astrand P, Rodahl K. Texbook of Work Physiology. New York: Mc Graw-Hill Book Company; 1985.

25. Stoudt H, Damon A, McFarlan R, Roberts J. Weight, height and selected body dimensions of adults. United States, 1960-62. US National Center for Health Statistics, Washington, DC: Government Printing Office; 1965.

26. Huerta R, Esparza J, Urquidez R. Validez de una ecuación basada en antropometría para estimar la grasa corporal en adultos mayores. ALAN 2007; 57 (4): 357-365.

28. Durnin J. Anthropometric methods for assessing nutritional status. En: Horwitz, Mac Fadyen, Munro, Srcrimshaw, Steen, Williams. Nutrition in the elderly. Oxford: Oxford University Press; 1989. p. 15-32.

29. Osorio J, Jiménez D, Vargas M, Apud E. Evaluation of physical load at work in high altitude mining. Proceedings IV World Congress on Mountain Medicine and high altitude Physiology; 2000 Oct. 1-6; Arica, Chile.

30. Apud E. An application of physiological 
anthropometry to a study of Chilean male workers. [Master's Thesis]. Inglaterra: Loughborough University; 1978.

31. Apud E. A human biological field study of Chilean forestry workers. [Doctoral thesis]. Inglaterra: Loughborough University; 1983.

32. Cumbre de Nutrición y Salud - Chile. El rol de la sociedad chilena en la prevención de la obesidad y otras enfermedades crónicas relacionadas con la dieta y la actividad física. Valparaíso: Ministerio de Salud; 2008.

33. Alonso F. La formación como variable estra- tégica para la prevención de riesgos laborales: el enfoque desde el INSHT. Ponencia presentada en la Mesa redonda: La calidad de la formación de los profesionales de la prevención. Universidad Internacional Menéndez Pelayo; 2002 sept 16-20; Santander, España.

34. Apud E, Meyer F. La importancia de la Ergonomía para los profesionales de la salud. Rev Ciencia y Enfermería. 2003; IX (1): 15-20.

35. Cobos D, Garí A. Necesidades de formación profesional en salud ocupacional: la percepción de los profesores de Madrid, España. Salud de los trabajadores. 2007; 15 (2): 99-106. 
